\title{
Conociendo el mundo de los polímeros: unidad didáctica como una estrategia para el aprendizaje
}

Recibido: 10-12-2010 | Aceptado: 28-12-2010

Knowing the world of polymers: Teaching unit as a learning strategy

\section{Martha Janneth Saavedra Alemán*}

Yolanda Ladino Ospina**

Leidy Moreno Rodriguez**

\begin{abstract}
Resumen: A través del diseño e implementación de una unidad didáctica que involucra actividades de organización y elaboración sobre la temática de polímeros, se pretende generar aprendizaje significativo mediante la relación existente entre el estudiante y lo cotidiano. La investigación parte del estudio de las ideas previas y el análisis del proceso de asimilación, para realizar posteriormente la diferenciación progresiva y reconciliación integradora sobre los nuevos conceptos.
\end{abstract}

\begin{abstract}
Through the design and implementation of a teaching unit that involves organization and development activities on the theme of polymers, is intended to generate significant learning by the students' relationship with the everyday situations. The research has as point of departure the study of previous ideas and the study of assimilation process to then make the progressive differentiation and integrative reconciliation on new concepts.
\end{abstract}

Palabras clave: unidad didáctica, polímero, aprendizaje significativo.
Keywords: Teaching unit, Polymer, Meaningful Learning.

\footnotetext{
* Departamento de Química, Universidad Pedagógica Nacional, Calle 72 No. 11-86. Bogotá, Colombia msaavedra@ pedagogica.edu.co.

** Departamento de Química, Universidad Pedagógica Nacional, Calle 72 No. 11-86. Bogotá, Colombia yladino@ pedagogica.edu.co.

*** Colegio Holístico Campestre. Cajicá, Cundinamarca. ikiruchang@hotmail.com
} 


\section{Introducción}

El conocimiento científico, tal como es enseñado en las aulas, sigue siendo ante todo un conocimiento verbal, en el que la tarea de los profesores consiste en dar la explicación, y la de los estudiantes, en el mejor de los casos, la de escuchar y copiar, lo que conlleva a que los educandos aprendan los conceptos por memorización, situación que ha generado en los sistemas educativos el replanteamiento de los objetivos en los procesos de enseñanza.

La educación en ciencias, específicamente la enseñanza de la química, es un área de conocimiento que ha estado sujeta a cambios constantes e innovadores, generando en los profesores la preocupación de cómo está siendo enseñada, lo que los ha llevado a proponer alternativas metodológicas novedosas como diseñar unidades didácticas para que el estudiante aprenda de manera significativa, vinculando la educación con el entorno cotidiano.

Dentro los problemas conceptuales que no han sido investigados, se encuentran los referentes a las concepciones que tienen los estudiantes en cursos de química orgánica, particularmente el tema de polímeros, esto debido quizá, a la complejidad de los conceptos que se estudian. En el caso particular, polímero y polimerización, en virtud de que muchos conciben los plásticos como la única aplicación material de polímero, lo que a su vez conlleva al desconocimiento de diferentes variedades de polímeros, como los naturales y sintéticos (Morrero, 2005). Lo anterior genera, de una u otra manera, problemas de aprendizaje y multiplicidad de concepciones alternativas en su mayoría erróneas; entre las más comunes se mencionan la utilización inadecuada del lenguaje científico para referirse a un mismo material, por ejemplo, en los libros de texto, se encuentran varias denominaciones de los polímeros, como plásticos, resinas, elastómeros y fibras sintéticas, presentando estos una única similitud a nivel macromolecular, pero sus propiedades son diferentes debido a su constitución química, lo cual conlleva a la utilización de una terminología útil, pero químicamente incorrecta (Zamora, 2006).

Por lo tanto en el presente trabajo se muestra una estrategia metodológica basada en el diseño e implementación de una unidad didáctica que contribuye al aprendizaje significativo de los conceptos asociados con el tema de los polímeros. Esta unidad didáctica fue aplicada a estudiantes de grado once del Colegio Santa Luisa, el cual pertenece a la Comunidad Jesuita de Bogotá.

\section{Criterios para planificación de unidades didácticas}

Para la elaboración de la unidad didáctica se tienen en cuenta los criterios establecidos por Sanmartí (2000):

- Criterio para la definición de objetivos/ finalidades: es importante tener presente que el objetivo de la unidad didáctica debe interrelacionar el profesor, el estudiante, la escuela y los problemas socio-ambientales.

- Criterio para la selección de contenidos: en este criterio es importante tener presente el tipo de contenidos, la relación que se puede generar entre la ciencia y la escolaridad y por último, es importante tener presentes los problemas ambientales y sociales que hacen parte del diario vivir de los estudiantes.

- Criterio para organizar y secuenciar los contenidos: se refiere a la selección de 
temáticas (simple-complejo), las cuales serán abordadas en la unidad didáctica para visualizarlas en el tiempo del cual se dispone.

- Criterios para la selección y secuenciación de las actividades: el proceso de aprender no es la actividad concreta, sino el conjunto de estas organizadas y secuenciadas, lo cual posibilita el flujo de interacciones entre el estudiantado y el profesorado; es por esto que las actividades no promueven un determinado conocimiento sino que plantean situaciones propicias para que los estudiantes actúen y sus ideas evolucionen en función de su situación personal (Perales. J, Cañal, P., 2000).

- Criterios para la selección y secuenciación de las actividades de evaluación: estas actividades deben ser consecuentes con todo el proceso realizado, ya que deben evaluarse conforme a los objetivos planteados al inicio de la unidad didáctica.

- Criterios para la organización y gestión en el aula: es importante crear entornos de aprendizaje que fomenten un adecuado ambiente de clase y unos valores favorables a la verbalización de las ideas y de las formas de trabajo, que promuevan los cambios de punto de vista, el respeto a todos ellos, su confrontación y la elaboración de propuestas.
El diseño de la unidad didáctica titulada Conociendo el mundo de los polímeros se muestra en la Tabla 1, la cual permitió la familiarización del estudiante con el concepto polímero, sus características y propiedades.

La unidad didáctica contiene una serie de actividades de elaboración (1, 3, 4, 11, $14,15)$ y de organización $(2,5,6,7,8,9,12$, $13,14,15)$, y los talleres 1, 2; las actividades complementarias hacen parte de los dos tipos de actividades.

Las actividades de elaboración integran y relacionan la nueva información con los conocimientos previos o ideas de anclaje, a partir de actividades de elaboración visual y verbal que permiten al estudiante tratar y codificar la información de manera más elaborada, porque atienden de manera básica su significado. Las actividades de organización permiten al estudiante hacer una reorganización constructiva de la información que ha de aprenderse, realizando procesos de agrupamiento o clasificación de los conceptos, con el objetivo de lograr una representación correcta del conocimiento.

En general estas estrategias fortalecen un proceso de descubrimiento y construcción de significados, enriqueciendo el aprendizaje significativo. 


\section{Ideas fundamentales}

1. El significado y uso que para la sociedad tienen los polímeros, tanto orgánicos como inorgánicos, y sus múltiples aplicaciones cotidianas.

2. La época actual es considerada la era de los polímeros.

3. A diario se utilizan diferentes tipos de materiales, desconociendo que se trata de polímeros.

\begin{tabular}{|c|c|c|c|}
\hline \multicolumn{4}{|c|}{ CONCEPTUALES } \\
\hline Contenidos & Objetivos & Actividades & Tiempo \\
\hline $\begin{array}{l}\text { Entrando al } \\
\text { mundo de los } \\
\text { polímeros. }\end{array}$ & $\begin{array}{l}\text { Comprender } \\
\text { el concepto } \\
\text { de polímero } \\
\text { diferenciándolo } \\
\text { de otro tipo de } \\
\text { sustancias. }\end{array}$ & $\begin{array}{l}\text { Actividad 1: dibujar objetos que sean } \\
\text { polímeros. } \\
\text { Actividad 2: se le presenta al } \\
\text { estudiante un cuadro descriptivo } \\
\text { con información acerca del papel, } \\
\text { las bolsas plásticas y llantas de hule; } \\
\text { a partir de estas descripciones el } \\
\text { estudiante debe determinar la unidad } \\
\text { monomérica y la clase de polímero a } \\
\text { la cual pertenece el objeto. } \\
\text { Actividad 3: identificar grupos } \\
\text { funcionales de una estructura } \\
\text { química. } \\
\text { Actividad 4: dibujar un ejemplo de } \\
\text { cada tipo de cadena características } \\
\text { de los polímeros. } \\
\text { Taller 1: resolver problemas de lápiz y } \\
\text { papel. Por ejemplo: } \\
\text { Los polímeros son la unión de } \\
\text { muchas moléculas pequeñas } \\
\text { llamadas monómeros que se repiten } \\
\text { constantemente formando una } \\
\text { gran cadena. Considerando que los } \\
\text { monómeros anteriores, por diferentes } \\
\text { reacciones de polimerización, forman } \\
\text { compuestos macromoleculares, } \\
\text { nómbrelos según la nomenclatura } \\
\text { sistemática de IUPAC. }\end{array}$ & 120 minutos \\
\hline
\end{tabular}




\begin{tabular}{|c|c|c|c|}
\hline Contenidos & Objetivos & Actividades & Tiempo \\
\hline $\begin{array}{l}\text { Un } \\
\text { acercamiento } \\
\text { a la historia de } \\
\text { los polímeros. }\end{array}$ & $\begin{array}{l}\text { Reconocer el } \\
\text { origen de la } \\
\text { ciencia de los } \\
\text { polímeros. }\end{array}$ & 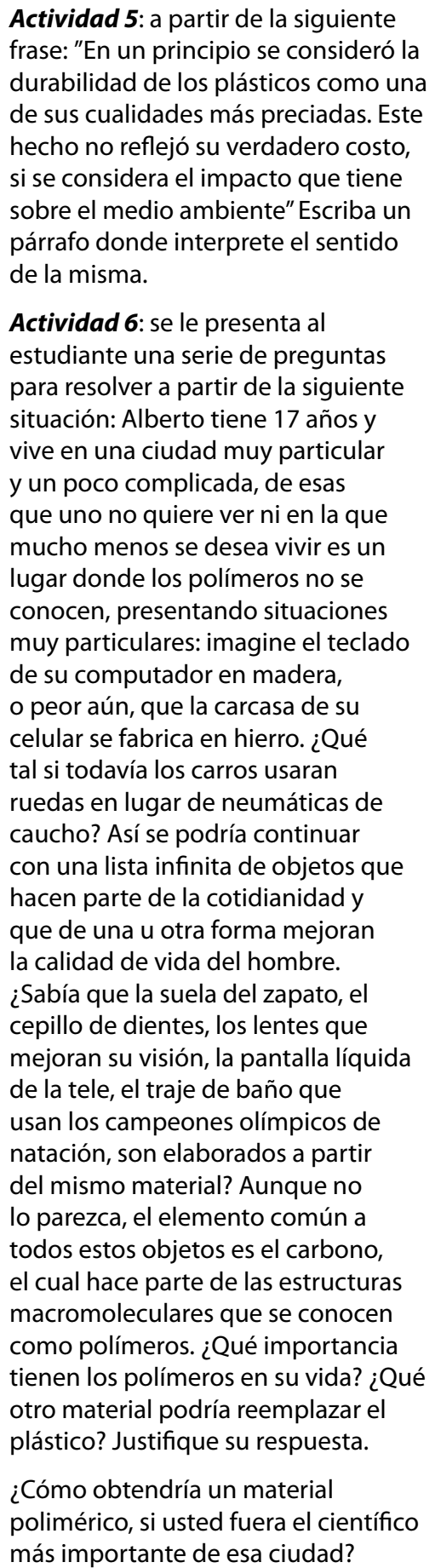 & 30 minutos \\
\hline
\end{tabular}




\begin{tabular}{|c|c|c|c|}
\hline Contenidos & Objetivos & Actividades & Tiempo \\
\hline $\begin{array}{l}\text { Distingamos } \\
\text { polímeros. }\end{array}$ & $\begin{array}{l}\text { Identificar y } \\
\text { diferenciar } \\
\text { los diferentes } \\
\text { tipos de } \\
\text { polímeros para } \\
\text { comprender } \\
\text { su naturaleza y } \\
\text { aplicación. }\end{array}$ & $\begin{array}{l}\text { Actividad 8: determinar la } \\
\text { clasificación de diferentes } \\
\text { polímeros. } \\
\text { Actividad 9: identificar el origen } \\
\text { de diferentes polímeros. }\end{array}$ & 45 minutos \\
\hline $\begin{array}{l}\text { Propiedades de } \\
\text { los polímeros. }\end{array}$ & $\begin{array}{l}\text { Reconocer la } \\
\text { elasticidad, } \\
\text { dureza y } \\
\text { resistencia } \\
\text { como } \\
\text { propiedades } \\
\text { mecánicas } \\
\text { de materiales } \\
\text { poliméricos. }\end{array}$ & $\begin{array}{l}\text { Actividad 11: indagar en } \\
\text { diferentes fuentes bibliográficas } \\
\text { las propiedades de varios } \\
\text { materiales poliméricos de uso } \\
\text { cotidiano. } \\
\text { Actividad 12: a partir de una lista } \\
\text { de materiales, reflexionar acerca } \\
\text { del uso de los polímeros. }\end{array}$ & 60 minutos \\
\hline $\begin{array}{l}\text { Obtención } \\
\text { de algunos } \\
\text { polímeros. }\end{array}$ & $\begin{array}{l}\text { Analizar y } \\
\text { diferenciar las } \\
\text { reacciones de } \\
\text { polimerización } \\
\text { que se utilizan } \\
\text { para la síntesis } \\
\text { de polímeros. }\end{array}$ & $\begin{array}{l}\text { Actividad 13: reconocer y } \\
\text { diferenciar a través de esquemas } \\
\text { las diferentes reacciones de } \\
\text { polimerización. } \\
\text { Taller 2: resolver ejercicios } \\
\text { de lápiz y papel y situaciones } \\
\text { problema de las reacciones } \\
\text { de obtención de compuestos } \\
\text { macromoleculares. Este taller esta } \\
\text { constituido por } 5 \text { ítems, donde } \\
\text { se manejan competencias para la } \\
\text { interpretación, argumentación y } \\
\text { proposición en cada uno de éstos. }\end{array}$ & 45 minutos \\
\hline $\begin{array}{l}\text { Los polímeros } \\
\text { que } \\
\text { constantemente } \\
\text { se usan. }\end{array}$ & $\begin{array}{l}\text { Relacionar los } \\
\text { conceptos, } \\
\text { métodos y } \\
\text { aplicaciones de } \\
\text { los polímeros } \\
\text { con la actividad } \\
\text { humana. }\end{array}$ & $\begin{array}{l}\text { Actividad 14: observar el video } \\
\text { Historia del plástico disponible } \\
\text { en: http://www.youtube.com/ } \\
\text { watch?v=qga-IM7CSnM y } \\
\text { responder una serie de preguntas. }\end{array}$ & 30 minutos \\
\hline
\end{tabular}




\begin{tabular}{|c|c|c|c|}
\hline \multicolumn{4}{|c|}{ PROCEDIMENTALES } \\
\hline $\begin{array}{l}\text { Analizar la } \\
\text { composición } \\
\text { macromolecular de } \\
\text { algunos polímeros. } \\
\text { Consultar diversas } \\
\text { fuentes de } \\
\text { información: libros, } \\
\text { videos, etc. }\end{array}$ & $\begin{array}{l}\text { Realizar } \\
\text { un mapa } \\
\text { conceptual que } \\
\text { esquematice } \\
\text { los conceptos } \\
\text { fundamentales } \\
\text { de la ciencia de } \\
\text { los polímeros. }\end{array}$ & $\begin{array}{l}\text { Actividad complementaria } \\
\text { 1: observar un video en la } \\
\text { dirección: http://es.youtube. } \\
\text { com/watch?v=ycUN7i74Thw y } \\
\text { realizar un resumen teniendo } \\
\text { en cuenta los conceptos de } \\
\text { polímero, molécula, proteínas, } \\
\text { carbohidratos, polietileno, } \\
\text { plástico, polimerización, } \\
\text { contaminación y efectos. } \\
\text { Actividad complementaria } \\
\text { 2: investigar qué polímeros } \\
\text { se utilizan en diversas } \\
\text { aplicaciones. } \\
\text { Actividad complementaria 7: } \\
\text { realizar un mapa conceptual } \\
\text { sobre la química de polímeros. }\end{array}$ & 90 minutos \\
\hline $\begin{array}{l}\text { Elaborar polímeros } \\
\text { y analizarlos } \\
\text { cualitativamente. } \\
\text { Explicar las } \\
\text { propiedades } \\
\text { mecánicas de } \\
\text { algunos materiales } \\
\text { poliméricos. }\end{array}$ & $\begin{array}{l}\text { Realizar } \\
\text { informes de } \\
\text { laboratorio } \\
\text { aplicando los } \\
\text { conceptos } \\
\text { aprendidos. }\end{array}$ & $\begin{array}{l}\text { Actividad 7: desarrollo de } \\
2 \text { prácticas de laboratorio } \\
\text { tituladas: } \\
\text { 1. Fabrique su propio polímero } \\
\text { sintético a partir de alcohol } \\
\text { polivinílico y borato de sodio. } \\
\text { 2. Fabrique su propio } \\
\text { biopolímero a partir de éter de } \\
\text { celulosa y alcohol polivinílico. } \\
\text { Actividad 10: reconocer las } \\
\text { propiedades mecánicas de } \\
\text { diferentes polímeros. } \\
\text { Actividad complementaria 4: } \\
\text { construcción y caracterización } \\
\text { de polímeros con clips de } \\
\text { diferentes colores. }\end{array}$ & 180 minutos \\
\hline
\end{tabular}




\begin{tabular}{|c|c|c|c|}
\hline \multicolumn{4}{|c|}{ ACTITUDINALES } \\
\hline $\begin{array}{l}\text { Identificar los } \\
\text { efectos del uso } \\
\text { frecuente de los } \\
\text { polímeros en la vida } \\
\text { cotidiana. }\end{array}$ & $\begin{array}{l}\text { Analizar el } \\
\text { impacto } \\
\text { ambiental de } \\
\text { los polímeros. }\end{array}$ & $\begin{array}{l}\text { Actividad 15: realizar un } \\
\text { resumen resaltando los } \\
\text { aspectos más relevantes } \\
\text { de la lectura Plásticos y } \\
\text { medio ambiente en: Revista } \\
\text { Iberoamericana de polímeros, } \\
\text { volumen } 3 \text { (2) Abril del } 2002 \text {. }\end{array}$ & 30 minutos \\
\hline $\begin{array}{l}\text { Potenciar el respeto } \\
\text { hacia el medio } \\
\text { ambiente. } \\
\text { Usar de manera } \\
\text { responsable, } \\
\text { los materiales } \\
\text { y reactivos en } \\
\text { las prácticas de } \\
\text { laboratorio. }\end{array}$ & $\begin{array}{l}\text { Promover } \\
\text { actitudes } \\
\text { favorables para } \\
\text { la conservación } \\
\text { del medio } \\
\text { ambiente } \\
\text { evitando el uso } \\
\text { del petróleo } \\
\text { como única } \\
\text { fuente de } \\
\text { obtención de } \\
\text { polímeros. }\end{array}$ & $\begin{array}{l}\text { Actividad 15: realizar un } \\
\text { resumen resaltando los } \\
\text { aspectos más relevantes } \\
\text { de la lectura Plásticos y } \\
\text { medio ambiente en: Revista } \\
\text { Iberoamericana de polímeros, } \\
\text { volumen } 3 \text { (2) Abril del } 2002 . \\
\text { Actividad 7: desarrollo de } \\
2 \text { prácticas de laboratorio } \\
\text { tituladas: } \\
\text { 1. Fabrique su propio } \\
\text { polímero sintético a partir de } \\
\text { alcohol polivinílico y borato } \\
\text { de sodio. } \\
\text { 2. Fabrique su propio } \\
\text { biopolímero a partir de } \\
\text { éter de celulosa y alcohol } \\
\text { polivinílico. }\end{array}$ & 90 minutos \\
\hline
\end{tabular}

Tabla 1. Diseño unidad didáctica Conociendo el mundo de los polímeros

\section{Caracteristicas metodológicas}

Esta investigación es abordada desde un enfoque cuantitativo con diseño experimental $y$, dentro de este, la subcategoría cuasiexperimental, que tiene como característica principal la manipulación de la variable independiente unidad didáctica, para observar su efecto y relación con una variable dependiente: aprendizaje significativo de los conceptos asociados a la temática de polímero, a partir del diseño y aplicación de una prueba de entrada (ideas previas) y una prueba final, para analizar la evolución del grupo antes y después de la implementación de la unidad didáctica.

El desarrollo de la investigación se basa en un conjunto de procesos sistemáticos y empíricos que se aplican al estudio de los polímeros y la enseñanza en el aula de esta temática (Hernández, 2006), usando la recolección de datos con base en la medición numérica y frecuencia de respuesta, 
mediante el uso de una matriz de datos, en la que se muestran los resultados de los instrumentos aplicados a los estudiantes con relación a las categorías, luego de ser expuestos a la implementación de la unidad didáctica, para así corroborar el aprendizaje significativo del tema polímeros a partir del desarrollo de actividades de elaboración y organización.

La metodología de investigación se llevó a cabo en cinco fases, a saber:

Fase 1: diseño y evaluación de la unidad didáctica, a cargo de tres expertos, que le permita al estudiante aprender significativamente.

Fase 2: diseño de todos los instrumentos con los que se recopiló la información necesaria para lograr el objetivo planteado en esta investigación.

Fase 3: aplicación de la unidad didáctica en las clases de química, con estudiantes del grado once $C$ del Colegio Santa Luisa. Para esta fase se dispone de 6 sesiones, cada una de 2 horas, en las cuales se incluye la aplicación de las pruebas inicial y final.

Fase 4: recolección de datos para evaluar el objetivo planteado.

Fase 5: se emiten conclusiones y recomendaciones que permiten comprender hallazgos, limitaciones y alcances sobre la investigación realizada.

Para medir los resultados obtenidos en la implementación de la unidad didáctica, se han establecido unos criterios de análisis, de acuerdo con los cuales serán analizadas cada una de las actividades y pruebas realizadas (Tabla 2).

\begin{tabular}{|l|l|l|}
\hline Código & Nivel & \multicolumn{1}{c|}{ Criterio } \\
\hline 3 & Alto & $\begin{array}{l}\text { Se evidencia un } \\
\text { trabajo en el que se } \\
\text { logra desarrollar un } \\
\text { aprendizaje significativo } \\
\text { completamente. }\end{array}$ \\
\hline 2 & Medio & $\begin{array}{l}\text { Se intenta realizar un } \\
\text { trabajo en el que se } \\
\text { logra desarrollar un } \\
\text { aprendizaje significativo } \\
\text { parcialmente. }\end{array}$ \\
\hline 1 & Bajo & $\begin{array}{l}\text { No se evidencia } \\
\text { ninguna característica } \\
\text { del aprendizaje } \\
\text { significativo. }\end{array}$ \\
\hline
\end{tabular}

Tabla 2. Niveles y criterios de valoración para cada aspecto del análisis

En la educación actual, todo lo que se enseña y lo que conciben los estudiantes con relación a una temática específica, tiene su origen en la escuela, en los medios de comunicación y en la vida diaria. De acuerdo con esta instancia, en la presente investigación se estudiarán tres aspectos implicados en las actividades, en correspondencia a las exigencias que se deben establecer en cualquier proceso educativo (Banet y Núñez, 1990).

De acuerdo con la propuesta de Banet y Núñez (1990), se plantean tres aspectos para evaluar el grado de aprendizaje significativo en las actividades establecidas en la unidad didáctica después de su aplicación. Estos aspectos se describen a continuación:

Grado de articulación. Se refiere a los conceptos que manifiestan cierto nivel de aislamiento o conocimientos memorísticos con una alta dependencia, o esquemas y redes conceptuales con determinado grado de amplitud, que agrupan y relacionan distintos conceptos. 
Nivel de aproximación al conocimiento científico. Considera nociones o ideas correctas que serán ampliadas durante el proceso de enseñanza-aprendizaje o, por el contrario, ideas erróneas o alternativas al conocimiento científico, que deben ser transformadas.

Relevancia. Se trata del uso de ideas básicas para comprender sus contenidos y relaciones con conceptos de otros temas, asignaturas o disciplinas, o bien, se refieren a aspectos más secundarios o de ampliación, por lo tanto entre mayor sea la cantidad de aspectos reconocidos, más alto será el desarrollo de este aspecto.

El análisis horizontal o simultáneo de estos tres aspectos permite introducir iniciativas para ampliar los conocimientos iniciales o plantear una reestructuración de los mismos, en caso de que sean erróneos.

\section{Resultados}

Los resultados obtenidos en la implementación de la unidad didáctica se tabularon a través de una matriz de datos, teniendo en cuenta los tres aspectos que evalúan el aprendizaje significativo (Banet y Núñez, 1990) presentados en la Tabla 3. En esta matriz, las filas corresponden a las actividades y las columnas a los aspectos establecidos para el análisis. Teniendo en cuenta estos parámetros, se procede a mirar el proceso de las actividades para establecer el aprendizaje significativo generado en los estudiantes de grado once del Colegio Santa Luisa (Ver Tabla 3).

Con los resultados expuestos, en primera instancia se determina que el nivel de articulación mostrado por los estudiantes a través del desarrollo de las actividades fue alto, criterio establecido en la Tabla 2, dejando claro que los estudiantes logran recono- cer los conceptos, dándoles un grado de importancia en el desarrollo de la temática, permitiendo que ellos los agrupen y los relacionen en la construcción de conocimiento y así adquieran un aprendizaje significativo.

Con relación al nivel de aproximación al conocimiento científico, se observa una disminución progresiva de las nociones correctas acerca de los conceptos enseñados. También se evidencia que las mayores dificultades se presentaron al desarrollar el taller 1, la actividad 9, y las actividades complementarias 2 y 3 .

Y por último, el nivel de relevancia, muestra que el desempeño por parte de los estudiantes a medida que se avanzaba en la aplicación de la unidad didáctica es alto, demostrando que comprenden y relacionan más conceptos con otros campos de acción, aprendiendo de manera subordinada y estableciendo una diferenciación progresiva en la estructura cognitiva.

Los resultados obtenidos en las pruebas inicial y final, permiten establecer una comparación respecto a la evolución conceptual de los estudiantes que se muestra en la Tabla 4, y la importancia de la aplicación de la unidad didáctica (Ver Tabla 3).

De acuerdo con los resultados mostrados en la tabla 4, se evidencia un cambio favorable en el desempeño, es decir, de desempeño medio-bajo a desempeño alto en los tres aspectos, que permiten evaluar el aprendizaje significativo. Estos cambios favorables son: en relación a la interpretación y reconocimiento de los conceptos asociados a la temática de los polímeros; la formulación de escritos coherentes y la relación de manera correcta y jerárquica de los conceptos en su estructura cognitiva; el reconocimiento de la importancia de los polímeros en la vida cotidiana del ser humano. 


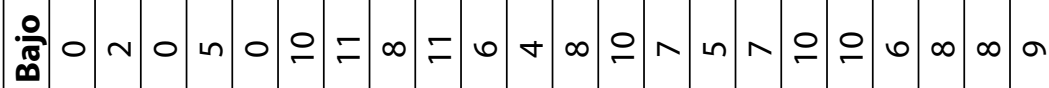

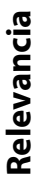

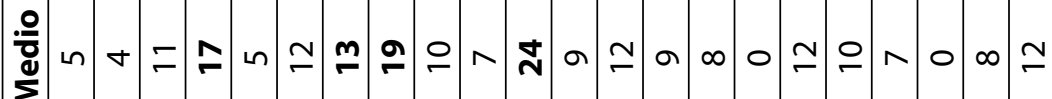

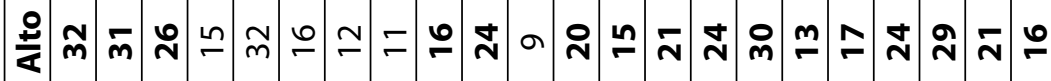

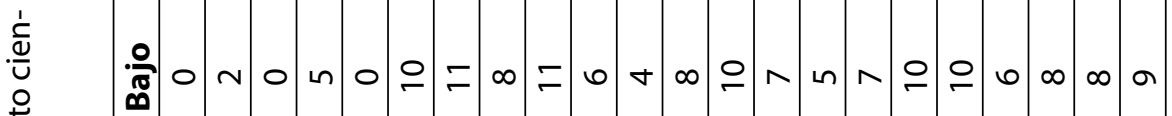

.

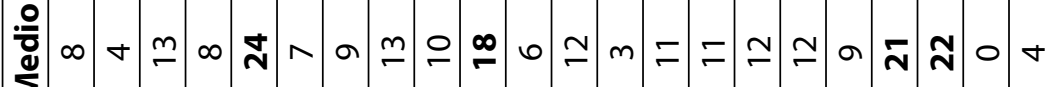

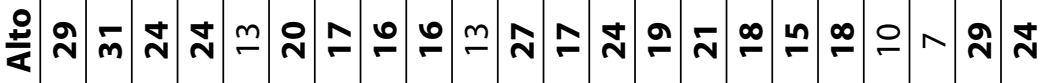

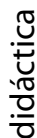

을

$\frac{}{4}$
$\frac{0}{2}$
$\frac{0}{2}$
$\frac{1}{0}$
$\frac{0}{0}$
$\frac{0}{0}$
$\frac{0}{0}$
$\frac{\pi}{0}$

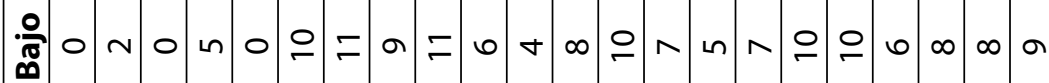
$\frac{3}{\frac{3}{0}}$

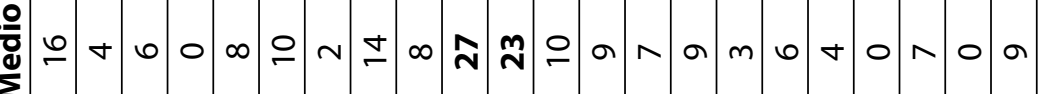

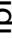

$\frac{\pi}{2}$

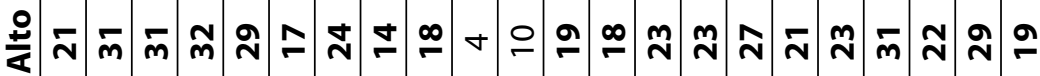

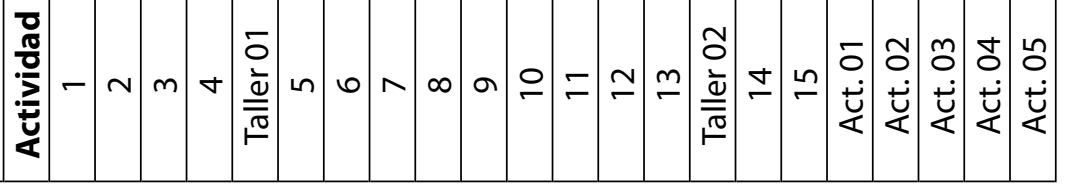




\begin{tabular}{|l|l|l|}
\hline \multicolumn{1}{|c|}{ Aspectos } & \multicolumn{1}{|c|}{ Prueba inicial } & \multicolumn{1}{c|}{ Prueba final } \\
\hline Grado de articulación & Desempeño medio & Desempeño alto \\
\hline $\begin{array}{l}\text { Grado de aproximación al } \\
\text { conocimiento científico }\end{array}$ & Desempeño bajo & Desempeño alto \\
\hline Grado de relevancia & Desempeño bajo & Desempeño alto \\
\hline
\end{tabular}

Tabla 4. Comparación resultados prueba inicial y prueba final

\section{Conclusiones}

La unidad didáctica diseñada para la enseñanza-aprendizaje de los conceptos asociados a la temática de polímeros, es una estrategia que involucró al estudiante de manera activa en el desarrollo de actividades de elaboración y organización, lo que muestra una evolución conceptual debido a que dieron respuesta a la gran mayoría de actividades con argumentaciones claras y coherentes.

Los estudiantes del grado once $C$, conocieron y reconocieron los polímeros como sustancias macromoleculares de gran aplicación en la cotidianidad. Desarrollando a cabalidad las actividades de elaboración y organización propuestas en la unidad didáctica, modificando las ideas y relacionando jerárquicamente los nuevos conceptos a través de asimilación. Los resultados mostraron que los estudiantes se apropiaron del tema, ya que identificaron su importancia en el desarrollo de la humanidad, estableciendo así las ventajas y desventajas que trae consigo el uso constante y desmesurado de ellos.

El análisis se realizó desde tres puntos de vista: articulación, aproximación al conocimiento científico y relevancia, lo cual permitió establecer qué, cómo, y cuántos conceptos iban asimilando a medida que se avanzaba en la unidad didáctica; se encontró que el grado de articulación que adquieren los estudiantes, se mantuvo en un nivel alto, en la mayoría de las actividades. El nivel de aproximación al conocimiento fue muy variado, encontrando dificultades en el taller 1, la actividad 9 y actividades complementarias 2 y 3, lo cual evidencia las formulaciones de nociones o ideas correctas. Con respecto a la temática, fue en general bueno y adecuado. En el nivel de relevancia, los estudiantes relacionaron casi todos los conceptos trabajados de manera correcta y jerárquica.

La construcción de esta unidad didáctica fortalece la aplicación de temáticas de uso cotidiano a la enseñabilidad de conceptos químicos en el aula, temáticas que no se tratan directamente en los currículos de química, sino de una manera transversal, permitiendo que los estudiantes identifiquen materiales de uso cotidiano y, a través de esto, asimilen nuevos conceptos químicos en su malla conceptual y adquieran un aprendizaje significativo

\section{Bibliografía}

Banet, E. y Núñez, F. (1990). Esquemas conceptuales de los alumnos sobre la respiración. En Enseñanza de las Ciencias, 8 (2), pp. 105-111. 
Hernández S. R., Fernández C. C. y Baptista L. P. (2006). Metodología de la investigación. Cuarta edición. México: McGraw Hill.

Moreno, J. (2005). Modelo constructivista en los procesos de enseñanza-aprendizaje: diseño de una unidad didáctica los plásticos. Revista electrónica de unidades didácticas y experiencias educativas IES Arico (1), pp. 25-35. Disponible en http://www.iesarico.es.

Perales, J. y Cañal, P. (2000). Didáctica de las ciencias experimentales. España: Editorial Marfil.

Perdomo, G. (2002). Plásticos y medio ambiente. En Revista Iberoamericana de Polímeros, 3 (2).
Sanmartí, N. (2000). El diseño de unidades didácticas, en Perales, P. y Cañal, P. (eds.). Didáctica de las ciencias experimentales. Alicante: Marfil.

Zamora, F. (2006). El alumno, el profesor y las macromoléculas. En Anales de la Real Sociedad Española de Química 102 (2), pp. 36-41.

Video: Historia del plástico. Disponible en: http://www.youtube.com/watch?v=qgaIM7CSnM. REALIZADA POR ENLACE VISUAL 2008 